L'iscrizione dovrà avvenire tramite compilazione della scheda di adesione disponibile sul nostro portale www.Odontoiatria33.it, che permetterà al provider di fornire via e-mail all'utente uno username e una password.

Per maggiori informazioni www.Odontoiatria33.it

\title{
Dalla malposizione all'inclusione del canino mascellare: diagnosi e previsione di eruzione
}

\section{From displacement to impaction of the maxillary canine: diagnosis and early eruption detection}

M.R. Ricchiuti๋, M. Mucedero, P. Cozza

Dipartimento di Ortodonzia, Università degli Studi di Roma Tor Vergata

\section{RIASSUNTO}

Obiettivi. Scopo del lavoro è illustrare le più recenti strategie di trattamento intercettivo in una fase di dentizione mista precoce nei pazienti con malposizione del canino permanente mascellare. Materiali e metodi. Dopo aver eseguito la diagnosi di dislocamento del canino è possibile attuare una serie di manovre di terapia intercettiva per favorire la sua eruzione. Sono descritte singolarmente tutte le metodiche di trattamento proposte dalla letteratura, specificando per ogni opzione l'apporto e la validità scientifica. Risultati. Da un'attenta analisi e revisione bibliografica emerge come l'approccio terapeutico nei confronti di una malposizione canina abbia subito un'importante evoluzione nel corso degli anni. Si passa infatti da un metodo di tipo passivo/ osservazionale, che prevede la semplice estrazione del canino deciduo e il monitoraggio radiografico, a interventi di terapia attiva più complessi che possono prevedere l'espansione del mascellare superiore, in associazione o meno alla distalizzazione dei settori latero-posteriori. Discussione. Nei casi di diagnosi precoce, il recupero del canino malposto prevede un nuovo approccio che integra le manovre già descritte con l'estrazione del canino e del primo molare decidui come elementi determinanti nella programmazione ortodontica. Conclusioni. L'obiettivo del trattamento intercettivo del canino mascellare è quello di aumentare le percentuali di successo di eruzione spontanea del canino permanente mascellare diagnosticato in una fase precoce di sviluppo, evitando meccaniche ortodontiche più complesse e biologicamente invasive per il paziente.

Parole chiave: Malposizione canina | Diagnosi precoce | Fattori di rischio | TC cone beam | Terapia intercettiva

\section{ABSTRACT}

Objectives. The aim of the study is to present the most innovative interceptive tre atment options during the early mixed dentition in subjects with a displaced maxillary permanent canine. Materials and methods. After a diagnosis of canine displacement, it is 
possible to apply several interceptive treatment options to encourage eruption. In this section, all the treatment approaches proposed by the literature have been individually described, and for each of them the relevant scientific validity has been assessed. Results. A careful literature review and analysis shows that the therapeutic approach of canine displacement has changed during the years and has passed from a passive/observational method, envisaging the extraction of the deciduous canine and the radiographic monitoring alone, to more complex protocols of interceptive treatment, that may include maxillary expansion, associated or not with the distalization of the latero-posterior segments. Discussion. In case of early diagnosis, the displaced canine recovery can be achieved through an innovative approach integrating the described protocols with the combined extraction of the deciduous canine and the first molar as determining factors of the orthodontic planning. Conclusions. The interceptive treatment of permanent maxillary canine displacement, when early diagnosed, aims to increase the prevalence rate of successful spontaneous eruption, while avoiding complex orthodontic and biologically invasive mechanics for the patient.

Key words: Canine displacement | Early diagnosis | Risk indicators | Cone beam computed tomography (CBCT)| Interceptive treatment

\section{Introduzione}

Dopo il terzo molare inferiore il canino mascellare è l'elemento più interessato da disturbi del tempo di eruzione e da anomalie di posizione [1].

Comunemente si possono distinguere due diverse situazioni cliniche: la malposizione e l'inclusione.

- La malposizione identifica una posizione anomala del canino all'interno dell'osso mascellare, prima del tempo normalmente previsto per la sua eruzione.

- L'inclusione è invece caratteristica di un elemento che si trova allinterno della compagine ossea con un apice radicolare chiuso, oltre il tempo fisiologicamente atteso per la sua comparsa in arcata [2,3].

La scoperta di un dente malposizionato o incluso è spesso casuale; queste anomalie infatti non sono accompagnate da dolore, disturbi o gonfiore. L'odontoiatra di base e/o l'odontoiatra pediatrico, durante una normale visita di controllo, possono evidenziare segni clinici di sospetto come la perdita precoce e asimmetrica di uno dei due canini decidui mascellari, un grave affollamento, la malposizione del laterale permanente ecc. $[4,5]$.

L'esperienza clinica unitamente alle conoscenze acquisite nel corso degli anni riguardo l'eziologia, la patogenesi, la capacità di lettura e interpretazione delle indagini radiografiche ci consentono oggi di individuare, in uno stadio di sviluppo precoce, una malposizione del canino mascellare e di intervenire per la correzione di quest'anomalia dentale. L'importanza della diagnosi e dell'intercettazione precoce del dislocamento del cani- no è associata non solo alla possibilità di evitare un approccio terapeutico più invasivo, semplificando le manovre ortodontiche, ma soprattutto a un più favorevole recupero fisiologico dell'elemento in arcata. II canino mascellare riveste infatti un ruolo fondamentale nell'equilibrio sia funzionale sia estetico del paziente: consente la disclusione dei denti posteriori nei movimenti di lateralità della mandibola, sostiene i tessuti molli, delimita la linea del sorriso, armonizza l'arcata superiore nel passaggio tra gli elementi posteriori e l'angolo delle commessure labiali [6].

\subsection{Epidemiologia}

I dati epidemiologici presenti in letteratura si riferiscono prevalentemente ai canini permanenti in inclusione mascellare; tale problematica nella dentatura decidua è molto rara [7].

La frequenza dell'inclusione oscilla tra lo 0,2\% e il 2,8\%, percentuali che variano nelle statistiche riportate da diversi autori in relazione al campione preso in esame e al tipo di studio effettuato. L'incidenza dell'inclusione del canino nei soggetti di sesso femminile è maggiore di circa due volte rispetto a quella del sesso maschile $[8,9]$. Nel 70-85\% delle inclusioni il canino è localizzato in posizione palatale, mentre nel restante 15-30\% è in posizione vestibolare o al centro della linea d'arcata [10].

\subsection{Eziopatogenesi}

Secondo Dewel [11] nessun elemento dentale è più interessante del canino mascellare dal punto di vista dello sviluppo. Questa osservazione è supportata non solo dal più lungo pe- 
riodo di formazione e calcificazione della sua gemma dentale, ma anche dalla complessa sequenza di movimenti che compie durante la migrazione dal punto in cui origina, intorno alla fine del primo anno di vita, fino al raggiungimento del piano occlusale tra i 9 e gli 11 anni di età [11]. II modello di sviluppo favorisce una più elevata ricorrenza di anomalie legata alla prolungata esposizione della bozza del canino a fattori ambientali, con la possibilità di una conseguente deviazione del suo tragitto di migrazione usuale [12]. Intorno ai 12 mesi di vita la corona del canino si trova fra le radici del primo molare deciduo, fra i 3 e i 4 anni passa verso la linea delle radici degli incisivi decidui, per poi accostarsi alla superficie vestibolare della radice del laterale. A 4 anni il primo molare deciduo e le gemme del primo premolare e del canino sono disposti in fila, orientati in direzione verticale. La crescita successiva della superfice vestibolare del mascellare fornisce spazio per consentire al canino il movimento in avanti fino a quando la sua cuspide entra in contatto con la superficie mesiale del corrispettivo deciduo.

Un'insufficiente presenza di spazio a livello della regione apicale, tale da non consentire il corretto allineamento degli incisivi laterali, fa sì che la corona del canino permanente eserciti una spinta sulla superficie distale dell'adiacente incisivo laterale. Si determina, pertanto, l'apertura fisiologica di un diastema conseguente all'anomala inclinazione dei laterali che caratterizza la fase cosiddetta del "brutto anatroccolo" ("ugly ducking"). La chiusura di questo diastema si verifica spontaneamente grazie allo scivolamento del canino permanente lungo la superficie distale del laterale contiguo.

Contemporaneamente la gemma del primo premolare prosegue il proprio cammino in direzione verticale portandosi più vicina al piano occlusale e al di sotto del canino permanente, erompendo in arcata prima di esso [13] (fig. 1). Tra i 5 e i 9 anni il movimento del canino permanente è orientato in direzione palatale e successivamente, dopo i 10 anni, tende a muoversi in direzione vestibolare [14].

La complessità del percorso di migrazione del canino mascellare, gli stretti rapporti di contiguità con gli elementi adiacenti, un'alterata sequenza di eruzione, la posizione anomala delle gemme dentarie e le anomalie a carico dell'adiacente incisivo laterale possono pertanto determinare una variazione dei rapporti tra la posizione delle gemme del canino permanente e del primo premolare o dell'incisivo laterale, risultante in un disturbo di eruzione.

Nonostante tutte le considerazioni sopra riportate, che individuano i fattori meccanici coinvolti nell'eziologia della malposizione canina, la letteratura rivolge grande attenzione anche ai fattori genetici [15]. La presenza simultanea di più anomalie dentali identifica, infatti, nel disturbo del canino solo un aspetto dei disordini generali di origine genetica. L'evidenza scientifica attesta che la malposizione del canino ricorre spesso in diversi membri della famiglia associandosi all'assenza congenita di altri elementi dentali, quali incisivi laterali e/o secondi premolari, colpendo maggiormente soggetti di sesso femminile. Questo dato suggerisce l'ipotesi genetica con un possibile coinvolgimento dei cromosomi sessuali $[16,17]$.

\section{Opportunità diagnostiche}

L'attenta analisi e la diagnosi dei disturbi di eruzione svolgono un ruolo fondamentale per consentire di programmare e pianificare un adeguato trattamento intercettivo. Infatti la mancata o non corretta valutazione di un canino potenzialmente incluso spesso può determinare gravi malposizioni che necessitano, per
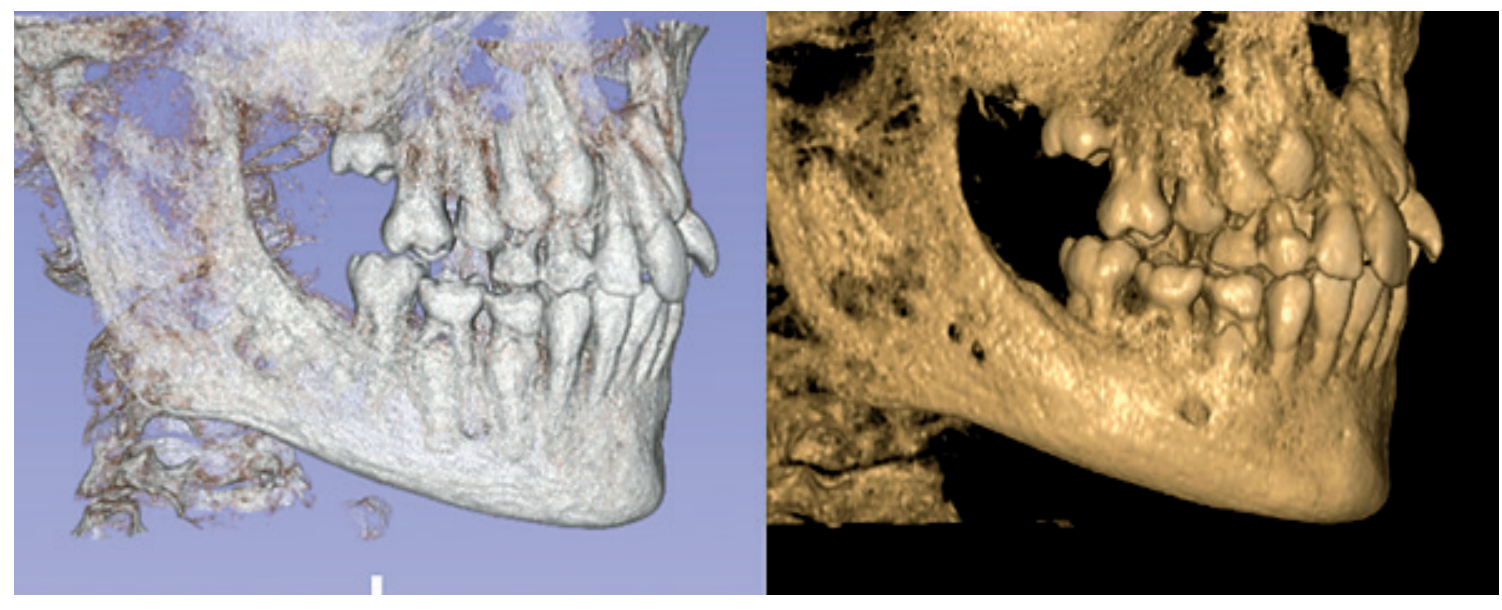

Fig. 1

Posizione

intraossea del canino in fase di eruzione durante il contatto della corona sulla superficie distale dell'adiacente incisivo laterale 
il recupero dell'elemento in arcata, l'applicazione di meccaniche ortodontiche complesse e biologicamente invasive, aumentando i costi della terapia e allungando i tempi di trattamento.

L'efficace riconoscimento e intercettamento della malposizione del canino mascellare richiedono pertanto una diagnosi iniziale precoce già intorno agli 8 anni di età, da effettuare esclusivamente attraverso indagini radiografiche che consentano di visualizzare la posizione intraossea dell'elemento considerato. Diversi studi infatti hanno individuato, quali indici radiografici, l'inclinazione e la localizzazione della cuspide del canino permanente non erotto (posizione della gemma del canino mascellare) rispetto alla radice dell'incisivo laterale erotto, come appare sull'ortopanoramica (OPT) in dentizione mista tardiva (10-13 anni) [18]. Tuttavia questi fattori radiografici possono essere usati per una valutazione diagnostica della dislocazione intraossea solo dopo i 10-11 anni di età e quindi in una fase che può essere definita "relativamente" precoce.

L'importanza della diagnosi precoce è associata alla posizione ectopica del canino già presente intorno ai 6 anni di età [14].

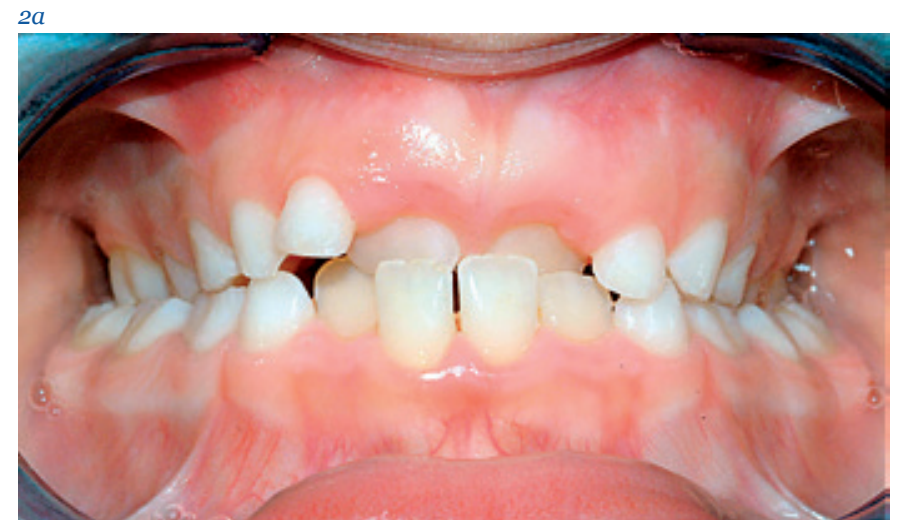

$\underline{2 c}$

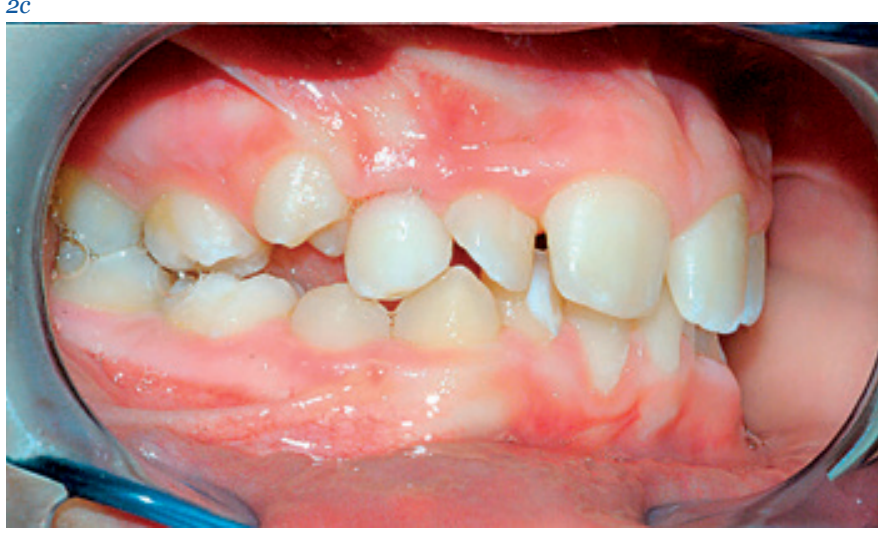

Sambataro et al. [19] hanno identificato nel telecranio in proiezione postero-anteriore un metodo diagnostico e prognostico al fine di riconoscere i disturbi di eruzione del canino superiore già intorno agli 8 anni.

Alla luce delle nostre conoscenze e della pratica clinica oggi siamo in grado di fare diagnosi precoce grazie sia all'esperienza raggiunta sia allo sviluppo di tecnologie di tomografia computerizzata con immagini tridimensionali in alta definizione eseguibili anche a basso dosaggio.

I segni clinici che possono indurre il sospetto di un dislocamento intraosseo sono numerosi e comprendono:

- una contrazione trasversale del mascellare;

- il cross-bite anteriore (fig. 2a,b);

- la perdita precoce di un canino deciduo associata alla mancata coincidenza delle linee mediane;

- la perdita precoce di entrambi i decidui con scomparsa dello spazio disponibile per mesializzazione dei settori posteriori;

una posizione anomala dell'incisivo laterale (fig. 2c,d);

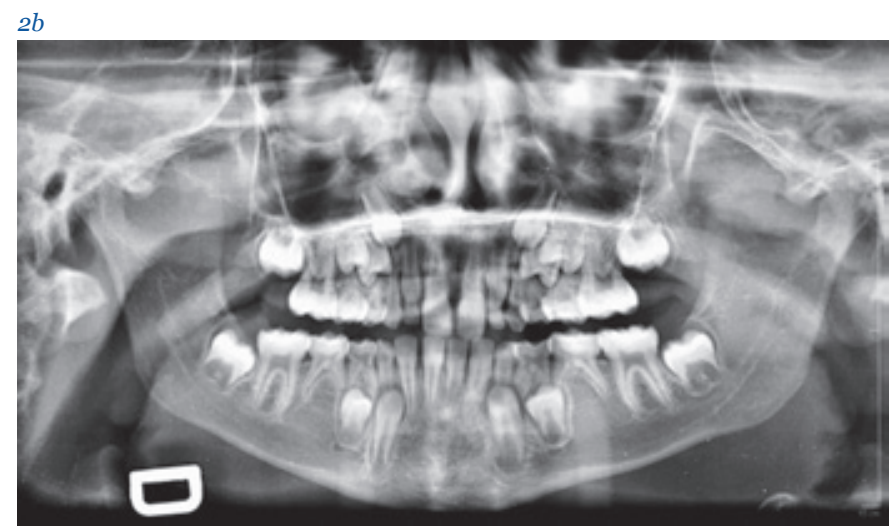

$2 d$

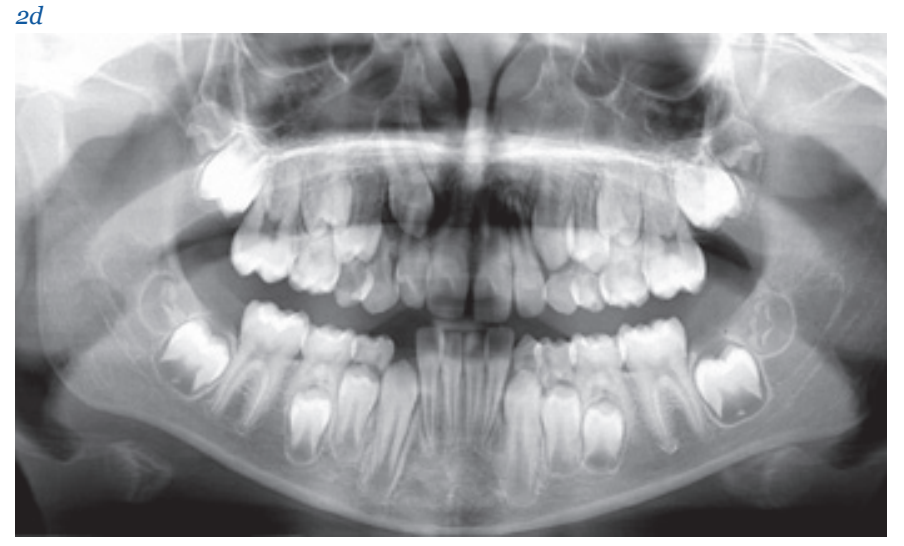

Fig. 2a-d a,b) Contrazione trasversale del mascellare superiore associata a cross-bite anteriore. Sull'OPT si notano: ritardo di formazione di 12 e 22 di forma conoide, agenesia di 35 e 45. c,d) Dall'esame clinico è possibile rilevare una posizione anomala dell'incisivo laterale permanente di destra. L'OPT evidenzia l'elemento 12 in malposizione intraossea 
- un'alterazione a carico della radice e/o della corona dell'adiacente incisivo laterale [20].

La persistenza di uno o entrambi i canini decidui in arcata, analogamente all'assenza della bozza canina alla palpazione sul lato vestibolare dell'alveolo, non può essere considerata segno clinico per la diagnosi precoce di malposizione intraossea del canino permanente. Infatti entrambe queste condizioni devono essere valutate a partire dai 9-10 anni di età, o ancora più tardi, in uno stadio di sviluppo del canino permanente più avanzato, quando la malposizione intraossea è ormai prossima all'inclusione e sta evolvendo verso una situazione di irreversibilità $[4,21]$.

La conferma di una malposizione, il cui sospetto sia stato indotto dal riscontro di uno o più dei segni clinici appena descritti, è supportata dagli esami radiografici.

L'OPT è la prima indagine cui il clinico ricorre per valutare gli aspetti anatomo-topografici del canino ed eventuali altre anomalie dentali associate o ostacoli all'eruzione, altrimenti non visualizzabili attraverso l'esame obiettivo. Molti autori hanno condotto le proprie ricerche fornendo una metodologia di lettura dell'OPT utile a identificare la posizione intraossea del canino e a individuare indici predittivi di inclusione discriminanti per la scelta terapeutica dell'ortodontista [3,8,18,22-26].

Tuttavia I'OPT, come le altre indagini radiografiche convenzionali, presenta un limite legato alla sovrapposizione delle strutture poste al di fuori della zona focale. Infatti la distanza non costante delle diverse strutture - in funzione della posizione del paziente, della presenza di malocclusione e di elementi posti in modo ectopico rispetto al piano del sensore - fa sì che la loro proiezione avenga con un ingrandimento variabile e imprevedibile [27]. Questo inconveniente rende difficile distinguere certi dettagli, soprattutto nel caso di denti malposizionati o inclusi quando le strutture differiscono anche solo leggermente in densità.

A oggi gli ostacoli della radiologia convenzionale bidimensionale sono stati superati dallintroduzione nella pratica clinica della tomografia computerizzata con ricostruzione 3D e dello scanner volumetrico cone-beam 3D (Cone Beam Computed Tomography, CBCT). La tridimensionalità è utile per diagnosticare la localizzazione e le complicanze di elementi malposizionati. L'innovazione introdotta con la CBCT offre una valida alternativa nell'ambito della diagnostica per immagini rispetto non soltanto ai radiogrammi dentali standard ma anche alla tomografia computerizzata medica tradizionale. Tali indagini sono ormai imprescindibili nella fase di pianificazione del trattamento per la scelta della metodologia da eseguire [28] (fig. 3).

\subsection{Segni e indici radiografici predittivi di malposizione}

Allo stato attuale la letteratura scientifica fornisce numerosi indici radiografici che consentono al clinico di effettuare una prima stima della posizione del canino mascellare anche attraverso la sola OPT. I parametri radiografici utilizzati per una valutazione prognostica dei canini in posizione anomala sono stati identificati per primi da Ericson e Kurol [18] e sono connessi alla probabilità di eruzione spontanea dei canini dislocati dopo estrazione del canino deciduo corrispondente al canino malposto.

- L'angolo $\alpha$ è dato dall'intersezione tra l'asse lungo del canino impattato e la linea mediana interincisiva.

- La distanza d rappresenta la distanza tra la cuspide del canino e il piano occlusale.

- Il settore s identifica l'area in cui si trova la corona del canino malposizionato.

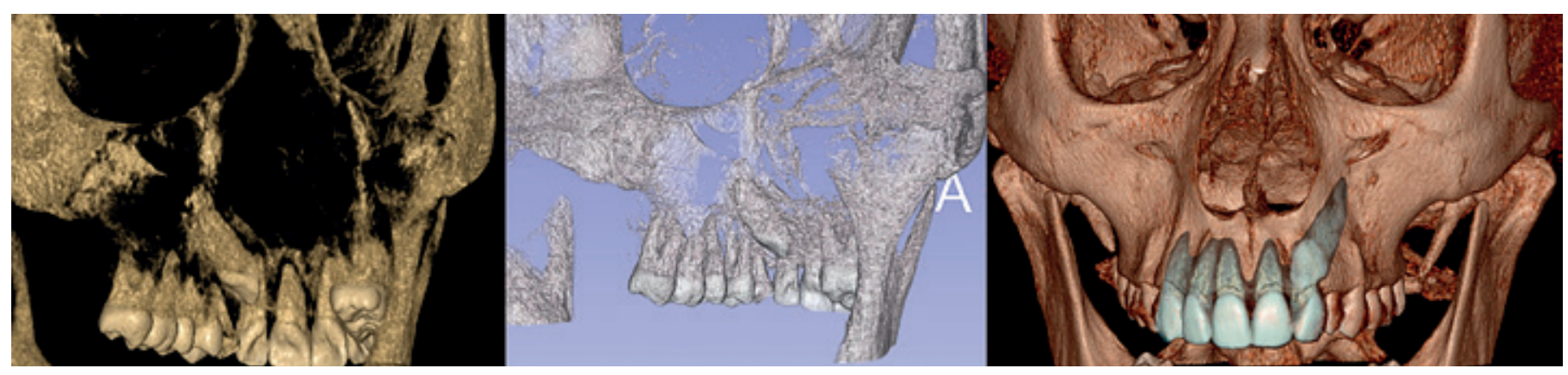

Fig. 3 CBCT: analisi dei dettagli per la visualizzazione della sede del 23 incluso e dei rapporti di contiguità con le radici degli elementi adiacenti 
Gli autori analizzano la normalizzazione del pattern di eruzione dei canini malposizionati attraverso la valutazione dei suddetti parametri radiografici a intervalli di 6, 12 e 18 mesi.

La metodologia di lettura dell'OPT proposta da Ericson e Kurol [18], successivamente modificata da Lindauer et al. [22], consente di prevedere l'inclusione del canino valutando la localizzazione della sua cuspide rispetto alla radice dell'incisivo laterale erotto durante la fase di dentizione mista. L'analisi descritta da Lindauer prevede una suddivisione della zona frontale in quattro settori: più la corona del canino è orientata mesialmente rispetto all'asse lungo dell'incisivo laterale, spostandosi dai settori più distali a quelli più mesiali, maggiori sono le probabilità di inclusione [22]. Sebbene numerosi altri autori $[3,23,24]$ abbiano proposto diverse misurazioni settoriali e angolari, la localizzazione del settore della cuspide del canino non erotto costituisce sicuramente il più importante fattore predittivo di un'eventuale inclusione, mentre l'angolazione non fornisce alcuna ulteriore significativa predicibilità [5].

In ogni, come è già stato sottolineato, queste variabili radiografiche assumono un significato diagnostico e prognostico della dislocazione intraossea in una fascia d'età più tardiva, ossia solo dopo i 10-11 anni. Gli unici ad aver introdotto un metodo diagnostico e prognostico atto a identificare i disturbi di eruzione in uno stadio di sviluppo precoce sono stati Sambataro et al. [19]. Gli autori individuano, nel telecranio in proiezione postero-anteriore, la distanza tra la corona del canino e il piano sagittale mediano come un fattore cruciale per la previsione precoce di inclusione: più la corona del canino è vicina alla linea mediana frontale all'età di 8 anni, maggiori sono le probabilità di mostrare un disturbo di eruzione [19].

Tutti gli studi presenti in letteratura sulla posizione dei canini dislocati hanno focalizzato l'attenzione sui rapporti tra la corona del canino malposto e le radici dei denti adiacenti posti mesialmente al canino stesso, ossia incisivi permanenti laterali e/o centrali.

Partendo dall'analisi dei rapporti anatomici che il canino contrae con il premolare durante il percorso intraosseo di eruzione [29] la nostra Scuola ha recentemente condotto una ricerca al fine di valutare l'associazione tra la mesioversione intraossea dei primi premolari mascellari e la dislocazione precoce dei canini, allo scopo di identificare un nuovo segno radiografico in dentizione mista precoce. La mesioversione del primo premolare mascellare (Mesially Displaced upper Premolar, MDP) è stata definita come l'inclinazione mesiale o la posizione mesiale del primo premolare rispetto al canino permanente in eruzio- ne. L'alta prevalenza di canini dislocati riscontrata nel gruppo di soggetti con una mesioversione del primo premolare rispetto al gruppo controllo suggerisce che la MDP sia un'anomalia dentale di posizione intraossea significativamente associata ai canini dislocati in dentizione mista. La MDP in presenza di un canino dislocato può essere considerata un nuovo indicatore radiografico di rischio di inclusione del canino mascellare [26]. Alla ricerca dei segni radiografici che consentono di porre diagnosi di malposizione in uno stadio precoce, in letteratura si ritrova l'associazione di altre anomalie dentali notoriamente riconosciute quali l'ipotrusione dei molari decidui, l'agenesia degli incisivi laterali superiori e/o dei secondi premolari, l'agenesia dei terzi molari, alterazioni a carico della radice degli adiacenti incisivi laterali $[16,17]$.

Nel 2010 Baccetti et al. [25] identificano l'inclinazione distale del secondo premolare mandibolare rispetto all'adiacente primo molare come un'anomalia dentale di sviluppo associata alla malposizione (Dislocazione Distale del Premolare, DDP). Tale inclinazione risulta anomala quando l'asse lungo della gemma del secondo premolare interseca il bordo mesiale del primo molare permanente. La distoinclinazione della gemma del secondo premolare è una condizione transitoria e solitamente si corregge spontaneamente quando il secondo premolare erompe in arcata. I risultati dello studio condotto dagli autori evidenziano che esiste una significativa associazione tra la DDP e il dislocamento palatale del canino mascellare; pertanto la DDP può essere considerata un indicatore di rischio di sviluppo per l'insorgenza della malposizione canina palatale [25]. È importante sottolineare l'aspetto clinico di questi segni radiografici e di tali associazioni di anomalie, in quanto nella maggior parte dei casi si manifestano cronologicamente prima che venga riconosciuta clinicamente la malposizione del canino permanente consentendo così di effettuare una diagnosi precoce e di adottare scelte terapeutiche intercettive più adeguate.

I metodi proposti per l'identificazione preventiva dei soggetti a rischio di inclusione del canino mascellare basati sui parametri radiografici possono essere coadiuvati in maniera vantaggiosa dal riconoscimento precoce di "segni di rischio" morfologici per l'inclusione del canino. È stato infatti dimostrato che i vari "disturbi dentali" - quali l'agenesia dell'incisivo laterale e/o del secondo premolare, la presenza di un incisivo laterale microdontico, conoide o agenesico, l'infraocclusione dei secondi molari decidui, l'ipoplasia dello smalto - concomitanti e ricorrenti nel singolo paziente possono essere riconosciuti come fattori associati a un'aumentata percentuale di inclusione del canino [16,17]. 
II professionista tuttavia non dovrà valutare tali indici in maniera isolata, ma nella loro globalità, tenendo presente che le informazioni ricavate non devono essere considerate in senso "assoluto" o come "elementi discriminanti" nella scelta di un piano di terapia. Infatti, come è già stato sottolineato, è opportuno avvalersi di esami strumentali più innovativi, come la CBCT, che grazie allo sviluppo di immagini tridimensionali consentono di valutare un'accurata e perfetta replica anatomica dei rapporti dell'elemento malposizionato con le strutture adiacenti [30].

\section{Possibilità terapeutiche: evoluzione dei tempi}

Nei soggetti che presentano un'evidente malposizione intraossea del canino mascellare, se un trattamento ortodontico non è intrapreso nel momento opportuno possono insorgere altre eventuali conseguenze quali il riassorbimento delle radici degli elementi permanenti contigui, formazioni cistiche [31] o il peggioramento della malposizione stessa che evolve in inclusione. Tale evenienza richiede un lavoro complesso, una gestione interdisciplinare con un aumento dei costi della terapia, della durata del trattamento e del rischio di comparsa di altri possibili effetti indesiderati.

Le procedure di terapia intercettiva sono invece in grado di ridurre l'incidenza dell'inclusione dei canini mascellari:

- evitando la necessità di un trattamento attivo in pazienti con un'occlusione accettabile;

- riducendo la necessità di un trattamento combinato ortodontico-chirurgico per il recupero e l'allineamento del canino in arcata;

- annullando il rischio di eventuali danni ai denti adiacenti causati dal canino malposto [8].

Nonostante l'esteso interesse sia per l'eziologia sia per la terapia dei canini dislocati, solo pochi studi negli ultimi vent'anni hanno focalizzato la loro attenzione sulle misure di terapia preventiva dell'inclusione canina [7,8,18,29,32-37].

\subsection{Estrazione del canino deciduo}

I primi protocolli clinici proposti includono l'estrazione dei corrispondenti canini decidui, con o senza procedure ortodontiche al fine di aumentare lo spazio disponibile nell'arcata mascellare.

Partendo dall'analisi della posizione di 46 canini ectopici palatali presenti in 35 soggetti di età compresa tra i 10 e i 13 anni, senza o con minima perdita di spazio, Ericson e Kurol [18] riportano che l'estrazione del canino deciduo, corrispondente al canino malposto, determina un cambiamento positivo della posizione nel 50\% dei casi già 6 mesi dopo l'estrazione; dopo 12 mesi tale percentuale raggiunge il 78\%. Gli autori, alla luce dei risultati ottenuti, suggeriscono tale procedura come trattamento di scelta in soggetti di 10-13 anni di età nei quali il canino permanente mascellare presenti una direzione di eruzione palatale.

Ericson e Kurol inoltre sottolineano che questo approccio non è indicato in età inferiore a 10 anni: prima di questo momento potrebbe infatti verificarsi una correzione spontanea della malposizione [18].

Risultati positivi, sebbene con percentuali di successo meno elevate di quelle ottenute da Ericson e Kurol [18], sono stati riportati da Power e Short [8] qualche anno dopo. Questi ultimi autori analizzano 39 pazienti di età compresa tra i 9 e i 14 anni, con 46 canini dislocati palatalmente, sottoposti a estrazione del canino deciduo corrispondente al permanente malposizionato. Dopo un monitoraggio sia clinico sia radiografico per un periodo massimo di 24 mesi, il 62\% dei canini malposti raggiunge una posizione eruttiva normale, mentre il 19\% mostra solo un'evoluzione positiva della sua posizione intraossea, confermando che il protocollo adottato consente al canino permanente, nella maggior parte dei casi, di migliorare la propria posizione. II valore aggiunto del lavoro di Power e Short [8] consiste nell'esame di diverse variabili, utili ai fini prognostici, da prendere in considerazione nella programmazione di tale procedura terapeutica.

La sovrapposizione orizzontale del canino all'adiacente incisivo laterale e la presenza di affollamento costituiscono i fattori di rischio più importanti. Infatti, sebbene l'affollamento non sia specificamente associato alla posizione del canino, la sua presenza - anche se moderata - può impedire la spontanea correzione del canino malposto, suggerendo la necessità di associare una procedura ortodontica volta a creare spazio [8].

\subsection{Estrazione del canino deciduo e terapia ortodontica fissa}

Nel 2002 Olive [33], partendo dai risultati ottenuti da Ericson e Kurol [18], conduce uno studio su un campione di 28 pazienti, tra gli 11 e i 16 anni di età, con 42 canini inclusi palatali: I'obiettivo dell'autore è risolvere l'anomalia di eruzione con il solo trattamento ortodontico. Olive riporta che nel 94\% dei casi la severità dell'inclusione si riduce dopo l'estrazione del canino deciduo associata al trattamento ortodontico, mentre nel 75\% dei casi il canino erompe in arcata tra i 2 e i 27 mesi dopo il recupero dello spazio con trattamento ortodontico di tipo fisso. 
Sebbene la terapia ortodontica risulti essere una procedura efficace per l'eruzione dei canini mascellari inclusi nei bambini tra gli 11 e i 15 anni, tuttavia questa scelta è raccomandata quando la cuspide del canino mascellare incluso non supera l'asse centrale della radice dell'incisivo laterale adiacente [33].

\subsection{Distalizzazione dei settori posteriori ed estrazione del canino deciduo}

L'intuizione di creare spazio nei casi di malposizione dei canini mascellari con affollamento moderato attraverso il movimento distale degli elementi dentali è stata discussa sia nello studio di Power e Short [8], nei primi anni Novanta, sia da Kuftinek e Sha- pira [38] qualche anno dopo; obiettivo era quello di ottenere un'eruzione spontanea dei canini.

Nel 2004 Leonardi et al. [34] propongono l'associazione della trazione extraorale all'estrazione del canino deciduo come approccio terapeutico intercettivo in grado di determinare con successo l'eruzione del canino permanente.

Nel 2008 Baccetti et al. [35] sottolineano come l'elevata percentuale di eruzione (87,5\%) ottenuta con l'associazione della trazione extraorale cervicale e l'estrazione del canino deciduo individui in queste due opzioni terapeutiche un nuovo e più efficace protocollo di terapia intercettiva [35]. L'impiego della trazione extraorale cervicale impedisce agli elementi dell'arca-

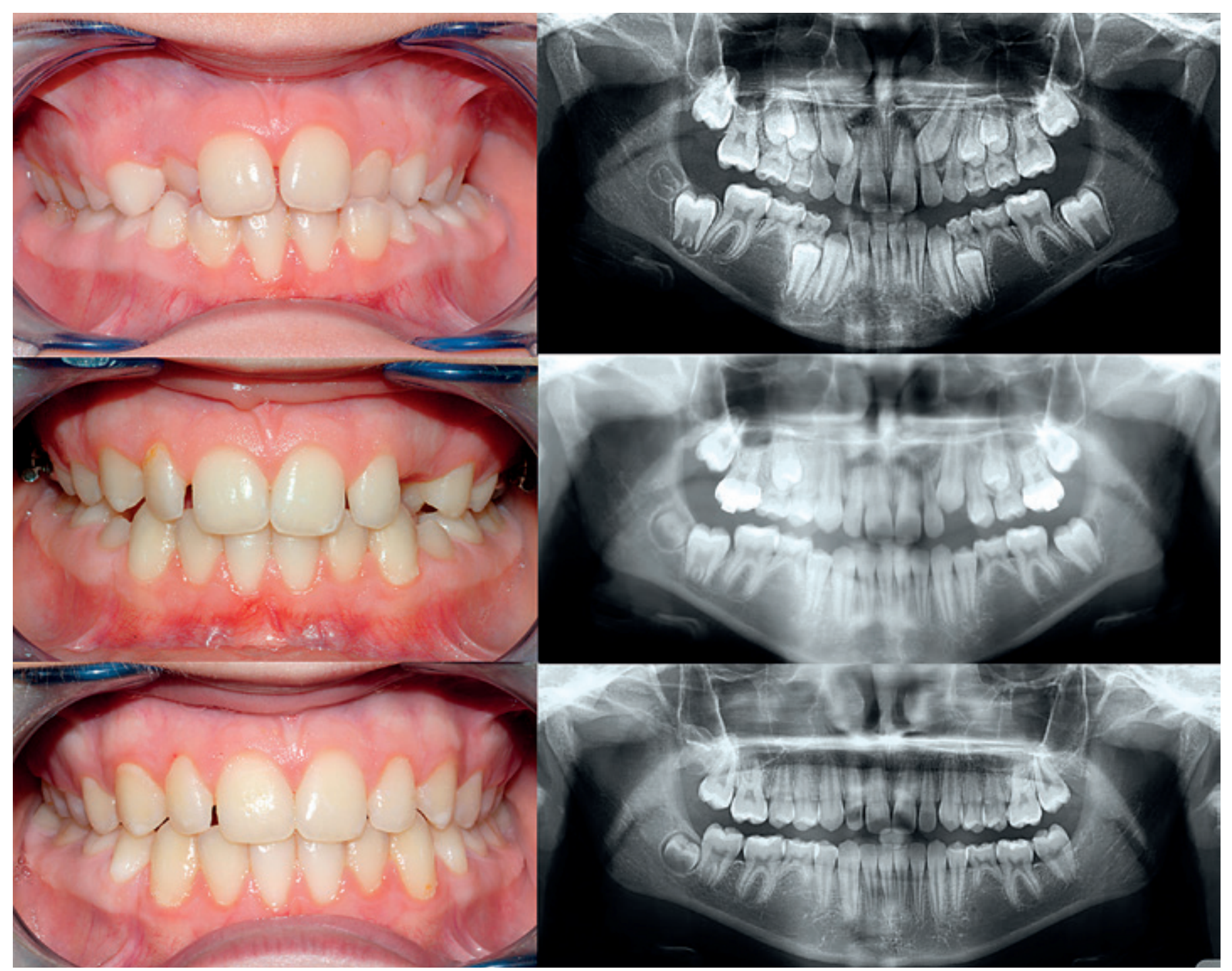

Fig. 4 Eruzione spontanea del canino permanente malposto ottenuta con l'associazione della trazione extraorale cervicale e l'estrazione del canino deciduo, senza terapia ortodontica fissa 
ta dentale superiore di muoversi mesialmente, mantenendo in tal modo lo spazio disponibile per l'eruzione del canino (fig. 4).

\subsection{Terapia ortopedica: espansione mascellare superiore}

La letteratura non è unanime nell'annoverare tra le cause di inclusione del canino mascellare un'ampiezza mascellare ridotta. Jacoby [10] sostiene che affollamento e lunghezza d'arcata inadeguati non sono associati al dislocamento palatale dei canini. Al contrario McConnell et al. [32] riportano che soggetti con inclusione del canino mascellare, senza alcuna distinzione di sede, mostrano un'ampiezza trasversale mascellare ridotta nella regione anteriore dell'arcata dentale; per tale motivo gli autori suggeriscono l'espansione ortopedica del mascellare come trattamento intercettivo della malposizione canina [32]. Schindel e Duffy [20] sottolineano che pazienti con una discrepanza trasversale sono più predisposti a manifestare l'inclusione del canino rispetto a pazienti senza problemi trasversali, raccomandando l'estrazione tempestiva del canino deciduo in associazione all'espansione ortopedica dell'arcata mascellare. Entrambe le manovre terapeutiche contribuiscono a fornire spazio per consentire un'adeguata eruzione del canino in arcata [20]. Mossi da vivo interesse verso l'individuazione di un protocollo più innovativo per l'intercettamento di tale anomalia di eruzione, Baccetti et al. [36] studiano gli effetti dell'espansione ortopedica del mascellare superiore in un gruppo di pazienti con malposizione palatale del canino, diagnosticata in uno stadio di sviluppo precoce, confrontandoli con un gruppo di controllo. La percentuale di successo di eruzione del canino dislocato dopo espansione rapida del mascellare superiore $(65,7 \%)$ è sovrapponibile a quella riportata da Ericson e Kurol [18] (78\%) e Power e Short [8] (62\%) dopo l'estrazione del canino deciduo, mentre è inferiore alla percentuale riportata da Baccetti et al. [36] (87,5\%) dopo distalizzazione dei settori posteriori associata a estrazione del canino deciduo. L'indicazione all'espansione ortopedica del mascellare è dettata dal dislocamento del canino e/o da una moderata discrepanza dento-alveolare associata a una contrazione dell'arcata mascellare rispetto all'arcata mandibolare. II vantaggio di tale approccio intercettivo consiste nell'intervento in epoca precoce, già intorno agli 8-9 anni di età (figg. 5 e 6).

\section{$>$ Terapia ortopedico-ortodontica}

a) Espansione del mascellare superiore e distalizzazione dei settori posteriori. Al fine di massimizzare gli effetti dei protocolli tera- peutici più efficaci presenti in letteratura, nel 2011 Armi et al. [39] hanno proposto un nuovo approccio terapeutico. L'obiettivo è quello di aumentare il diametro trasversale dell'arcata superiore attraverso l'espansione del mascellare (Rapid Maxillary Expander, RME) e mantenere e/o incrementare la lunghezza d'arcata mediante la distalizzazione dei settori posteriori. I risultati ottenuti dal presente lavoro suggeriscono che l'associazione dell'espansione con la trazione extraorale cervicale riconosce un potenziale di successo maggiore rispetto a quello ottenuto attraverso il solo approccio ortopedico (85,7\% vs 65,7\%).

Tale metodica non considera la possibilità di associare l'estrazione del canino deciduo corrispondente al canino malposizio-
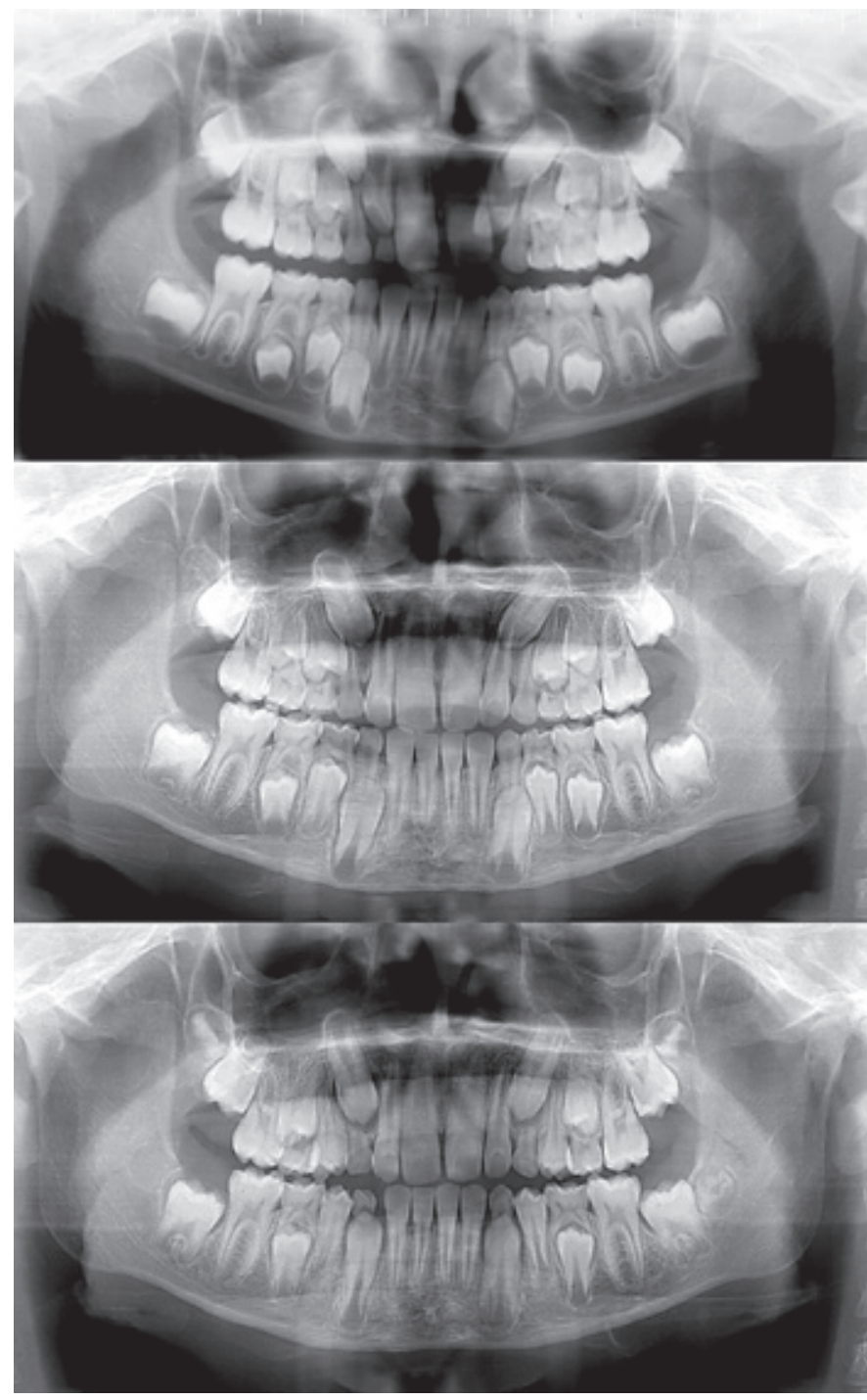

Fig.5 L'OPT evidenzia eruzione spontanea del canino malposto dopo espansione del mascellare 


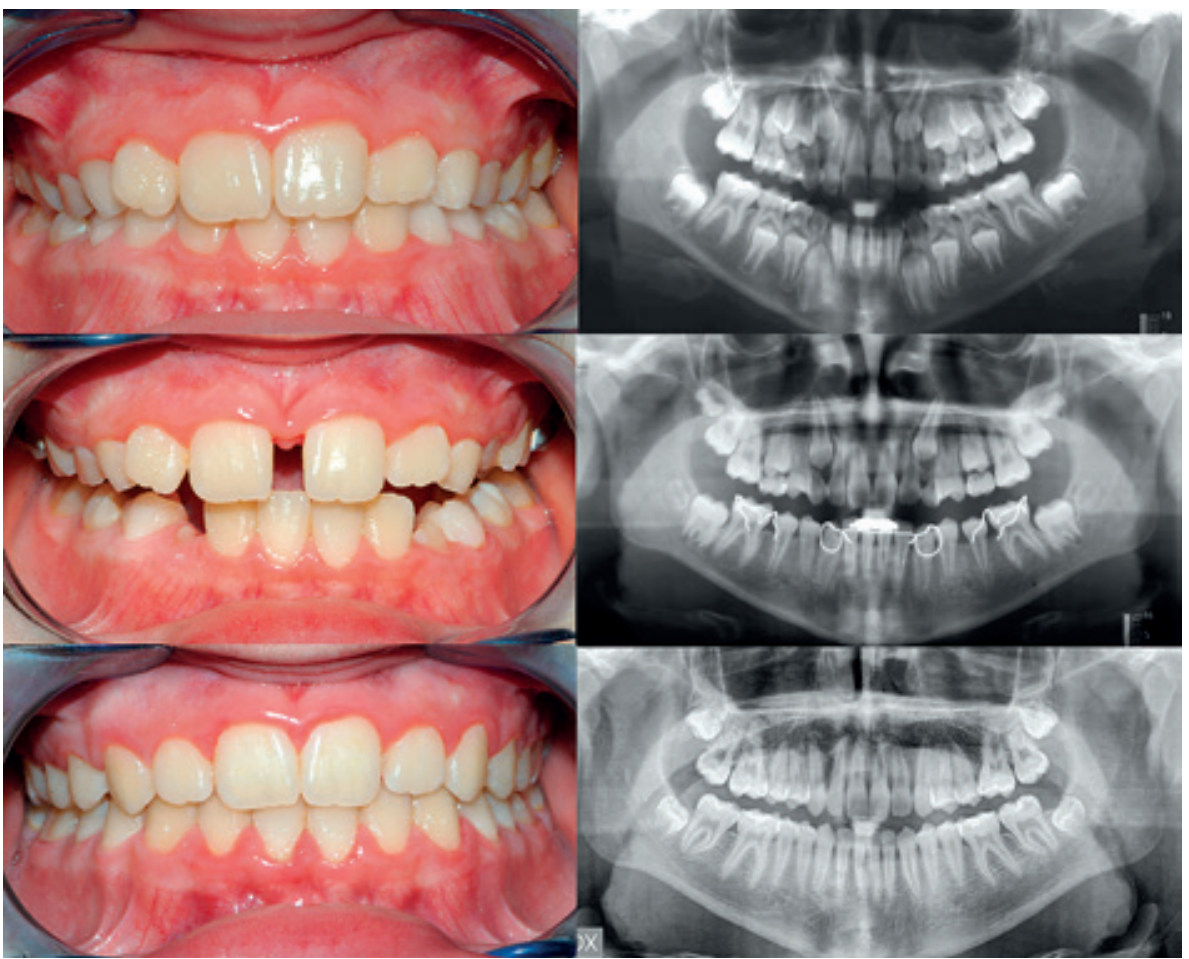

Fig. 6 Eruzione spontanea del canino permanente malposto ottenuta dopo espansione ortopedica del mascellare superiore

nato, in quanto il mantenimento del canino deciduo in arcata può risultare molto utile nel caso in cui si renda necessario un intervento combinato ortodontico-chirurgico per la disinclusione del canino, quando l'evoluzione della malposizione diventa sfavorevole. La presenza del canino deciduo consente infatti di eseguire la tecnica del tunnel indicata per garantire un suc-

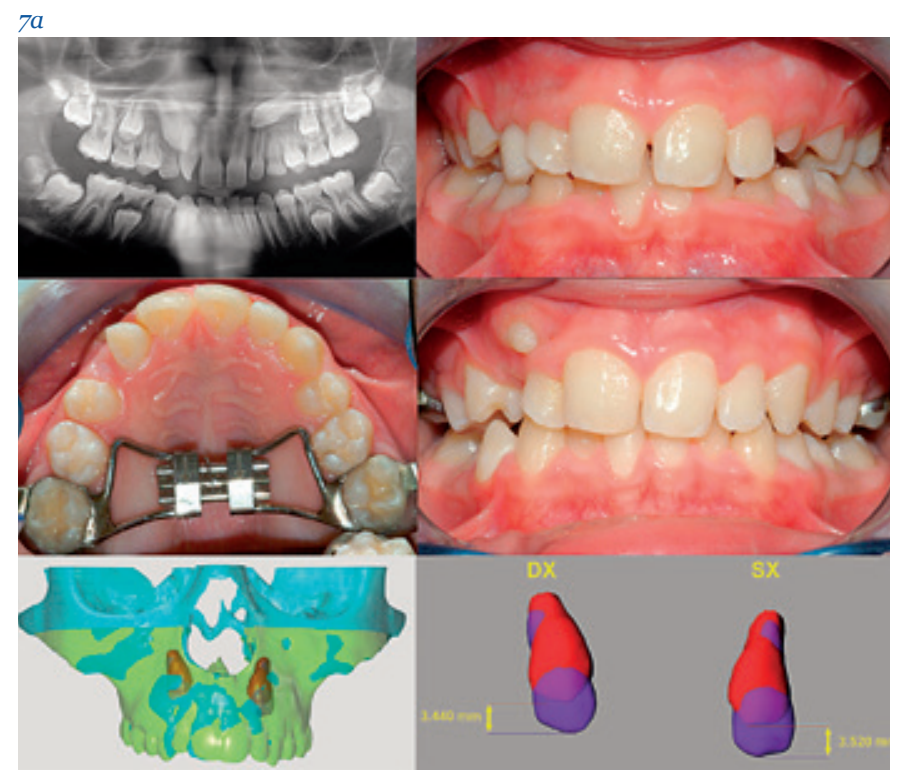

cesso parodontale ottimale del canino riposizionato a lungo termine (fig. 7a,b).

b) Espansione del mascellare superiore, ancoraggio dei settori posteriori, estrazione del canino deciduo. Nel corso degli ultimi anni la ricerca scientifica ha accolto nuove proposte terapeutiche

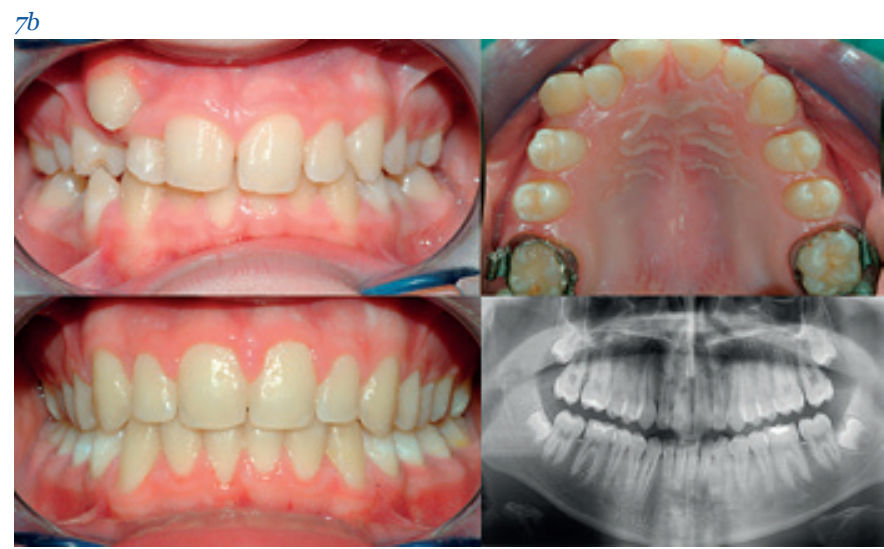

Fig. 7a,b Espansione del mascellare superiore e distalizzazione dei settori posteriori. Le immagini sono state elaborate con software per la pulizia e segmentazione delle strutture anatomiche, l'assemblaggio e la sovrapposizione delle due mascelle prima (To) e dopo (T2) l'espansione e con software per le misurazioni 
di intercettamento della malposizione del canino mascellare. Ł̀ stato presentato un nuovo protocollo costituito da espansione rapida del mascellare, estrazione del canino deciduo e applicazione di un arco transpalatino, impiegato dopo una diagnosi di malposizione canina in fase di dentizione mista tardiva [40]. Gli autori sostengono che, all'estrazione del canino deciduo, l'associazione dell'espansione rapida del mascellare e dell'arco transpalatino in una fascia d'età più avanzata (10-13 anni) aumenti la percentuale di successo di eruzione sino al 90\%. Tale percentuale risulta notevolmente superiore anche rispetto ai valori riportati da Ericson e Kurol [18] dopo la semplice estrazione del canino deciduo (65\%) eseguita sempre nellintervallo 10-13 anni.

L'espansione del mascellare viene effettuata con lo scopo primario di migliorare il processo di eruzione dei canini malposizionati in presenza di altre indicazioni ortodontiche quali un affollamento medio o moderato delle arcate dentali o la tendenza a una malocclusione di Classe II o III [40].

\subsection{Terapia estrattiva non ortodontica}

Da un'analisi delle proposte terapeutiche presenti in letteratura - come più volte sottolineato - il comune denominatore dei più efficaci approcci intercettivi ortodontici risulta essere l'estrazione del canino deciduo corrispondente al canino in malposizione intraossea palatale. Nei casi in cui la persistenza del canino deciduo costituisce un ostacolo di natura meccanica per l'eruzione del corrispettivo permanente, la sua estrazione è in grado di ricondizionare il fisiologico processo intraosseo di eruzione fino alla sua emergenza in arcata. Tuttavia non è stato ancora spiegato l'esatto meccanismo attraverso il quale tale manovra terapeutica determina un'evoluzione favorevole nella malposizione canina.

Alessandri Bonetti et al. [29] propongono un nuovo protocollo terapeutico associando all'estrazione del solo canino deciduo l'estrazione del primo molare deciduo adiacente al fine di massimizzare i vantaggi di queste due procedure estrattive, in soggetti di età compresa tra gli 8 e i 13 anni. Intorno all'età di 9 anni si presenta il momento più critico per il canino, in quanto la sua corona esegue una curva in direzione mesiale verso la linea mediana, per poi cambiare nuovamente direzione con un movimento orizzontale della cuspide distalmente verso il primo premolare. È stato valutato il pattern di eruzione dei canini malposizionati in un periodo di osservazione di circa 18 mesi attraverso l'analisi dei parametri radiografici individuati da Ericson e Kurol [18].
La percentuale di successo riportata nel gruppo di soggetti sottoposti al nuovo protocollo introdotto da Alessandri Bonetti et al. [29] (97,3\%), rilevata in termini di miglioramento della posizione intraossea del canino dislocato e di eruzione, supera nettamente la percentuale ottenuta nel gruppo che ha ricevuto la semplice estrazione del canino deciduo (78,6\%). II meccanismo che spiega i risultati ottenuti è legato al fatto che, in aggiunta ai vantaggi dell'estrazione del canino deciduo già descritti, la rimozione del primo molare deciduo accelera l'eruzione e promuove l'uprighting del primo premolare stimolando una correzione della posizione del canino permanente.

\subsection{Nuovi orizzonti: un approccio di terapia intercettiva combinata ortopedico-estrattivo- ortodontica}

Alla luce delle possibilità terapeutiche proposte e descritte in letteratura e della nostra esperienza clinica oggi suggeriamo un approccio intercettivo combinato ortopedico-estrattivo-ortodontico. Alla base vi è un'attenta fase diagnostica resa possibile grazie alla conoscenza dei tempi di permuta degli elementi dentali, al riconoscimento precoce della malposizione stessa e alla capacità di lettura degli esami diagnostici. Tale protocollo, in presenza delle indicazioni cliniche opportune, prevede:

- una prima fase di espansione ortopedica del mascellare superiore, anche in epoca precoce (7-9 anni), in grado di gestire lo spazio intraosseo del canino permanente;

- l'associazione dell'estrazione del canino e del primo molare decidui per promuovere l'eruzione del primo premolare e stimolare la correzione della posizione intraossea del canino permanente in eruzione;

- una seconda fase ortodontica di distalizzazione dei settori posteriori volta a stimolare e migliorare la posizione del canino inducendone l'uprighting.

Il momento in cui è possibile porre una diagnosi precoce può essere già intorno ai 7 anni. L'OPT consente di visualizzare e di riconoscere i segni clinici ma anche radiografici ormai notoriamente associati alla malposizione del canino mascellare. Le indagini radiografiche ripetute a intervalli di 12 mesi devono inevitabilmente coadiuvare l'esame obiettivo al fine di compiere un monitoraggio diligente degli elementi in eruzione, con particolare attenzione ai canini mascellari. L'obiettivo fondamentale della diagnosi precoce è quello di intercettare e normalizzare le discrepanze scheletriche, sviluppare un'adeguata dimensione dell'arcata per favorire la corretta eruzione dei denti permanenti e migliorare la funzione occlusale (fig. 8a,b). 
$8 a$

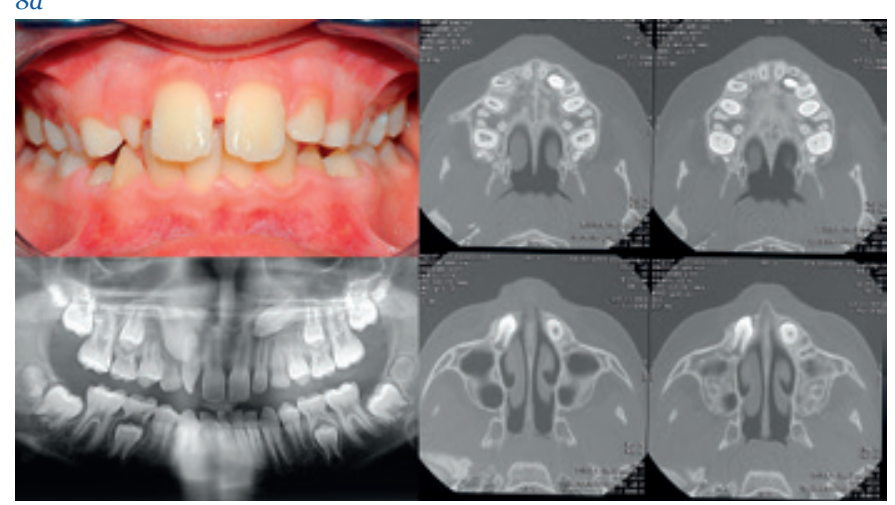

Fig. 8a,b Un approccio di terapia intercettiva combinata ortopedico-ortodontico-estrattiva. Spontaneo recupero del 13 in arcata dopo espansione e distalizzazione, aggancio chirurgico del 23 e trazione in arcata

\section{Quali le motivazioni di un trattamento ortodontico precoce?}

L'importanza della diagnosi e del trattamento ortodontico precoce è dettata dalla possibilità di ridurre l'incidenza di inclusione dei canini in malposizione, di evitare eventuali estrazioni di elementi permanenti non programmate, di scongiurare la possibilità di dover ricorrere all'intervento chirurgico per consentire l'aggancio del canino per il suo riposizionamento in arcata, di diminuire il rischio di insorgenza di riassorbimenti e complicanze a carico degli elementi contigui (tab. I).

\section{Conclusioni}

Nel presente articolo sono illustrate scelte terapeutiche ed espresse considerazioni cliniche derivate dall'esperienza personale degli autori nel trattamento intercettivo e chirurgicoortodontico di numerosi casi con canini superiori malposizionati e/o inclusi. Sono presi in esame diversi fattori diagnostici e terapeutici e descritte in modo particolareggiato le tecniche intercettive ortodontiche utilizzate (tab. I).

Gli approcci terapeutici suggeriti dalla letteratura per la risoluzione della malposizione canina raccomandano di intervenire in una fase di dentizione mista tardiva (10-12 anni). Tuttavia la nostra esperienza clinica evidenzia che, intervenendo ancor prima degli 8 anni per risolvere problematiche ortopedico-or-

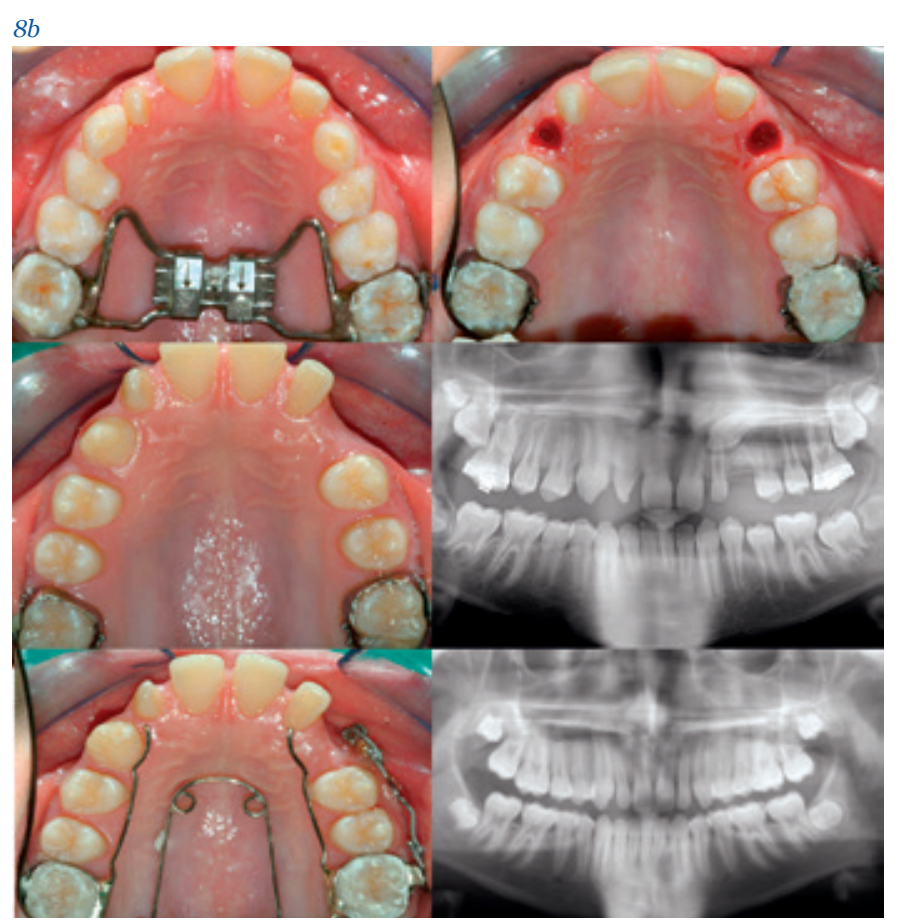

\section{Tab.I Dalla malposizione all'inclusione del canino mascellare: scheda riassuntiva}

- Le basi per una diagnosi precoce

- Conoscenza dell'anatomia e della permuta

- Riconoscimento dei segni clinici predittivi

- Possibilità di eseguire controlli periodici

- Gli obiettivi di un trattamento precoce

- Eliminazione dei fattori predisponenti la malposizione

- Intercettamento e normalizzazione delle discrepanze scheletriche

- Sviluppo di un'adeguata dimensione dell'arcata per favorire la corretta eruzione dei denti permanenti

- Miglioramento della funzione occlusale

- I cardini di un trattamento precoce

- Esame clinico

- Indagine radiografica

- Monitoraggio diligente della permuta

- Controlli periodici fino alla completa eruzione di tutti gli elementi permanenti in arcata

I benefici del trattamento ortodontico precoce

- Riduzione del rischio di inclusione

- Possibilità di evitare meccaniche ortodontiche complesse

- Possibilità di evitare procedure biologicamente invasive

- Possibilità di evitare il rischio d'insorgenza di complicanze

- Possibilità di evitare estrazioni di elementi permanenti non programmate

- La scelta della metodologia terapeutica

- Estrazione del canino deciduo

- Estrazione del canino deciduo + Distalizzazione dei settori posteriori

- Espansione del mascellare superiore

- Espansione del mascellare superiore + Distalizzazione dei settori

posteriori \pm Estrazione del canino

- Estrazione del canino deciduo + Estrazione del primo molare deciduo

- Espansione del mascellare + Estrazione del canino e del primo molare decidui + Distalizzazione dei settori posteriori 


\section{Box 1 Linee guida}

I concetti e le nozioni esposti nel presente lavoro possono essere così schematizzati, in modo da fornire i concetti chiave necessari per affrontare e gestire una situazione clinica complessa.

\section{Prima degli 8 anni di età}

- Controlli periodici per il monitoraggio della permuta.

- Qualora necessario in base all'esame clinico: OPT al fine di individuare la presenza di eventuali anomalie dentali.

- In assenza di alterazioni della permuta, problematiche ortopedicoortodontiche, anomalie dentali o altro, il soggetto viene monitorato una volta l'anno per controllare se la sequenza di eruzione di tutti gli elementi dentali avviene in maniera fisiologica.

- Al contrario, il riscontro di problematiche trasversali e/o sagittal (quali il cross-bite laterale o anteriore) associate o meno alla presenza di affollamento guiderà il clinico verso un intervento di terapia intercettiva ortopedico-ortodontica.

\section{Tra gli 8 e gli 11 anni di età}

Individuazione di eventuali fattori di rischio di malposizione: - contrazione del mascellare superiore in associazione o meno alla presenza di affollamento;

- inclinazione disto-vestibolare degli incisivi laterali permanenti (tilting degli incisivi laterali);

- alterazioni di forma e dimensioni degli incisivi laterali permanenti (conoidi, microdontici);

- ipotrusione dei molari decidui, sospetta agenesia degli incisivi laterali, ipoplasia dello smalto degli incisivi centrali superiori o dei primi molari permanenti [25];

- assenza della bozza canina alla palpazione (sul lato vestibolare dell'alveolo al di sopra del corrispondente deciduo in arcata);

- In presenza di uno o più dei segni clinici sopra elencati sarà necessario approfondire la valutazione diagnostica attraverso un percorso radiologico completo (OPT, LL, PA) volto a:

- localizzare la posizione del canino e i suoi rapporti con gli elementi adiacenti;

- utilizzare gli indici radiografici predittivi di malposizione (agenesia degli incisivi laterali, ipotrusione dei molari decidui, dislocazione distale delle gemme dei secondi premolari inferiori, mesioversione delle gemme dei primi premolari superiori) $[25,38,39]$;

- identificare i fattori prognostici di inclusione (localizzazione del canino nel settore, distanza centro corona canino-piano sagittale mediano); - programmare un adeguato piano di trattamento.

- Se è presente la malposizione del canino, prima di procedere alla programmazione terapeutica è opportuno richiedere un'indagine radiografica 3D (CBCT).

- Solo dopo aver valutato la posizione intraossea del canino e i suo rapporti con gli elementi contigui si procederà alla scelta di un adeguato piano di trattamento intercettivo (tab. I).

\section{Tra gli 11 anni e i 13 anni di età}

- Analisi della condizione di malposizione. L'esame clinico e le indagini radiografiche potranno rivelare una malposizione del canino permanente in associazione o meno:

- alla persistenza del canino deciduo

- alla presenza di un grave affollamento;

- a un ritardo di permuta.

In questi casi il piano di trattamento, oltre a prevedere una delle metodiche elencate in tabella I, dovrà considerare l'eventualità di una programmazione chirurgica.

\section{Dopo i 14 anni di età}

- La diagnosi di canino malposto/incluso dopo i 14 anni di età presenta una prognosi più sfavorevole riguardo la possibilità di recupero in arcata attraverso il solo trattamento intercettivo. Tuttavia una fase ortopedica di espansione mascellare può essere necessaria per la risoluzione di un'eventuale contrazione mascellare e per il controllo dello spazio in arcata in presenza di affollamento.

- La programmazione terapeutica prevedrà pertanto un approccio ortodontico-chirurgico rivolto alla gestione coerente dello spazio in arcata, alla scelta dell'ancoraggio e alla programmazione dei movimenti di eruzione. todontiche già manifeste quali cross-bite anteriore e/o laterale, Classi III o altre malocclusioni con priorità di trattamento, è possibile ottenere nello stesso tempo un miglioramento della malposizione intraossea del canino mascellare.

La descrizione di tutte le metodiche esposte nel presente lavoro ha la finalità di illustrare le alternative terapeutiche a disposizione del clinico in caso di diagnosi di malposizione intraossea del canino mascellare.

L'attenta valutazione delle caratteristiche occlusali individuali del paziente, il grado di collaborazione, I'analisi della posizione intraossea dell'elemento malposizionato e i rapporti con gli elementi contigui, la conoscenza e l'esame delle eventuali alterazioni presenti a livello della regione apicale dell'area incisale e premolare guideranno la scelta del clinico verso la metodica più opportuna per ottenere il successo dell'eruzione.

In tutti i casi in cui si ha l'opportunità di visitare pazienti in età precoce è possibile scegliere in modo ragionato il momento più idoneo e l'approccio terapeutico più adeguato per intercettare o risolvere la malposizione del canino mascellare. L'applicazione di uno dei protocolli descritti (box 1) nei soggetti con dislocamento del canino è fortemente suggerita nei casi che presentano le indicazioni cliniche necessarie; infatti in assenza di tali indicazioni l'ortodontista valuterà la scelta di altre opzioni terapeutiche per la risoluzione del singolo caso.

\section{CONFLITTO D'INTERESSI}

Gli autori dichiarano di non avere alcun conflitto di interessi di ordine economico o di altro tipo.

\section{FINANZIAMENTI ALLO STUDIO}

Gli autori dichiarano di non aver ricevuto finanziamenti per il presente studio.

\section{CONSENSO INFORMATO}

Per i casi clinici inseriti i pazienti hanno sottoscritto il consenso informato.

\section{BIBLIOGRAFIA}

1. Moyers RE. Handbook of Orthodontics. 4th Ed. Chicago: Yearbook Medical Publishers, 1988; pp. 235-40.

2. Kokich VG. Surgical and orthodontic management of impacted maxillary canines. Am J Orthod Dentofacial Orthop 2004;126(3):278-83.

3. Baccetti T, Crescini A, Nieri M, Rotundo R, Pini Prato GP. Orthodontic treatment of impacted maxillary canines: an appraisal of prognostic factors. Prog Orthod 2007;8(1):6-15.

4. Becker A. Trattamento ortodontico dei denti inclusi. Torino: UTET, 1998.

5. Cozza P, Mucedero M, Ricchiuti MR, Baccetti T. Il canino superiore incluso. Diagnosi e terapia basate sull'evidenza scientifica. Testo atlante. Bologna: Martina, 2010 
6. Okeson JP. Management of temporomandibular disorders and occlusion. 6th Ed. St. Louis (MO); Toronto: Elsevier Mosby, 2007.

7. Kuftinek MM, Shapira Y. The impacted maxillary canine: I. Review of concepts. ASDC J Dent Child 1995;62(5):317-24.

8. Power SM, Short MB. An investigation into the response of palatally displaced canines to the removal of deciduous canines and an assessment of factors contributing to favourable eruption. $\mathrm{Br}$ J Orthod 1993;20(3):215-23

9. Sacerdoti R, Baccetti T. Dentoskeletal features associated with unilateral or bilateral palatal displacement of maxillary canines. Angle Orthod 2004;74(6):725-32

10. Jacoby $\mathbf{H}$. The etiology of maxillary canine impactions. Am J Orthod 1983;84(2):125-32

11. Dewel BF. The upper cuspid: its development and impaction. Angle Orthod 1949;19:79-90.

12. Rozylo-Kalinowska I, Kolasa-Raczka A, Kalinowski P. Dental age in patients with impacted maxillary canines related to the position of the impacted teeth. Eur J Orthod 2011;33(5):492-7.

13. McSherry PF. The ectopic maxillary canine: a review. Br J Orthod 1998;25(3):209-16

14. McSherry P, Richardson A. Ectopic eruption of the maxillary canine quantified in three dimensions on cephalometric radiographs between the ages of 5 and 15 years. Eur J Orthod 1999;21(1):41-8.

15. Chalakkal P, Thomas AM, Chopra S. Displacement, location, and angulation of unerupted permanent maxillary canines and absence of canine bulge in children. Am J Orthod Dentofacial Orthop 2011;139(3):345-50

16. Baccetti T. A controlled study of associated dental anomalies. Angle Orthod 1998;68(3):267-74

17. Peck S, Peck L, Kataja M. Prevalence of tooth agenesis and peg-shaped maxillary lateral inicisor associated with palatally displaced canine (PDC) anomaly. Am J Orthod Dentofacial Orthop 1996;110(4):441-3.

18. Ericson S, Kurol J. Early treatment of palatally erupting maxillary canines by extraction of the primary canines. Eur J Orthod 1988;10(4):283-95.

19. Sambataro S, Baccetti T, Franchi L, Antonini F. Early predictive variables for upper canine impaction as derived from posteroanterior cephalograms. Angle Orthod 2005;75(1):28-34.

20. Schindel RH, Duffy SL. Maxillary transverse discrepancies and potentially impacted maxillary canines in mixed-dentition patients. Angle Orthod 2007;77(3):430-5

21. Ericson S, Kurol J. Longitudinal study and analysis of clinical super vision of maxillary canine eruption. Community Dent Oral Epidemiol 1986;14(3):172-6.

22. Lindauer SJ, Rubenstein LK, Hang WM, Andersen WC, Isaacson RJ. Canine impaction identified early with panoramic radiographs. J Am Dent Assoc 1992;123(3):91-7.

23. Stivaros N, Mandal NA. Radiographic factors affecting the management of impacted upper permanent canines. J Orthod 2000;27(2):169-73.

24. Warford JH Jr, Grandhi RK, Tira DE. Prediction of maxillary canine impaction using sectors and angular measurement. Am J Orthod Dentofa- cial Orthop 2003;124(6):651-5

25. Baccetti T, Leonardi M, Giuntini V. Distally displaced premolars: A dental anomaly associated with palatally displaced canines. Am J Orthod Dentofacial Orthop 2010;138(3):318-22

26. Mucedero M, Ricchiuti MR, Franchi L, Cozza P. Association between mesially displaced upper premolars and early displaced canines. Eur J Paed Dent 2015. In press.

27. Duterloo HS. Atlante della dentizione dellinfanzia. Diagnosi ortodontica e radiologia panoramica. Scienza e tecnica dentistica. Milano: Edizioni Internazionali, 1992.

28. Chen Y, Duan P, Meng Y, Chen Y. Three-dimensional spiral computed tomographic imaging: a new approach to the diagnosis and treatment planning of impacted teeth. Am J Orthod Dentofacial Orthop 2006;130(1):112-6

29. Alessandri Bonetti G, Incerti Parenti S, Zanarini M, Marini I. Double vs single primary teeth extraction approach as prevention of permanent maxillary canines ectopic eruption. Pediatr Dent 2010:32:407-11.

30. Alqerban A, Jacobs R, Fieuws S, Willems G. Comparison of two cone beam computed tomographic systems versus panoramic imaging for localization of impacted maxillary canines and detection of root resorption. Eur J Orthod 2011;33(1):93-102.

31. Ericson S, Kurol PJ. Resorption of incisors after ectopic eruption of maxillary canines: a CT study. Angle Orthod 2000;70(6):415-23.

32. McConnell TL, Hoffman DL, Forbes DP, Janzen EK, Weintraub NH. Maxillary canine impaction in patients with transverse maxillary deficiency. ASDC J Dent Child 1996;63(3):190-5

33. Olive RJ. Orthodontic treatment of palatally impacted maxillary canines. Aust Orthod J 2002;18(2):64-70.

34. Leonardi M, Armi P, Franchi L, Baccetti T. Two interceptive approaches to palatally displaced canines: a prospective longitudinal study. Angle Orthod 2004;74(5):581-6.

35. Baccetti T, Leonardi M, Armi P. A randomized clinical study of two interceptive approaches to palatally displaced canines. Eur J Orthod 2008;30(4):381-5.

36. Baccetti T, Mucedero M, Leonardi M, Cozza P. Interceptive treatment of palatal impaction of maxillary canines with rapid maxillary expansion: a randomized clinical trial. Am J Orthod Dentofacial Orthop 2009;136(5):657-61.

37. Alessandri Bonetti G, Zanarini M, Incerti Parenti S, Marini I, Gatto MR. Preventive treatment of ectopically erupting maxillary permanent canines by extraction of deciduous canines and first molars: A randomized clinical trial. Am J Orthod Dentofacial Orthop 2011;139(3):316-23.

38. Kuftinek MM, Shapira Y. The impacted maxillary canine: II. Clinical approaches and solutions. ASDC J Dent Child 1995;62(5):325-34.

39. Armi P, Cozza P, Baccetti T. Effect of RME and headgear treatment on the eruption of palatally displaced canines: a randomized clinical study. Angle Orthod 2011;81(3):370-4.

40. Baccetti T, Sigler LM, McNamara JA Jr. An RCT on treatment of palatally displaced canines with RME and/or a transpalatal arch. Eur J Orthod 2011;33(6):601-7. 